\title{
Origen y Distribución de los Ramos Motores del Nervio Mediano para los Músculos Superficiales de la Región Anterior del Antebrazo en Individuos Brasileños
}

\author{
Origin and Distribution of the Motor Branches for the Superficial Muscles \\ of the Anterior Region of the Forearm in Brazilian Individuals
}

Riveros, A."; Sousa-Rodrigues, C.F.** \& Olave, E.***

RIVEROS, A.; SOUSA-RODRIGUES, C. F. \& OLAVE, E. Origen y distribución de los ramos motores del nervio mediano para los músculos superficiales de la región anterior del antebrazo en individuos brasileños. Int. J. Morphol., 34(1):356-364, 2016.

RESUMEN: El nervio mediano es el encargado de la inervación de los músculos del plano superficial del compartimiento anterior del antebrazo, para ello, da origen a lo largo de su trayecto en el brazo y antebrazo, una serie de ramos para estos músculos. Algunos ramos se originan de troncos comunes $(\mathrm{Tc})$. Con el objetivo de obtener una descripción anatómica y biométrica del origen y distribución de los ramos del nervio mediano en los músculos superficiales del antebrazo: pronador redondo (Pr), palmar largo (PL), flexor radial del carpo (FRC), flexor superficial de los dedos (FSD), así como clasificar a estos ramos de acuerdo a su origen, se estudiaron 30 miembros superiores de cadáveres de individuos brasileños, adultos fijados en formaldehído al $10 \%$, realizando disecciones y registrando sus orígenes en relación a la línea biepicondilar (LBE). Con los resultados obtenidos, se realizó una clasificación de los ramos para los músculos del plano superficial del compartimiento anterior en 4 patrones (I, II, III, IV; el I dividido en IA y IB) incluyendo también, el origen del nervio interóseo anterior (NIA). En el patrón I A (29\%), los ramos se originaron de proximal a distal en la siguiente secuencia: TcPr, PL, FRC, NIA, R1FSD, R2FSD; el patrón I B (41 \%) mostró TCPr, TcFRC y PL, NIA, R1FSD, R2FSD; en el padrón II (4 \%) fue TcPr y FRC, PL, NIA, R1FSD, R2FSD, R3FSD; en el padrón III (11 \%) fue TcPr, Tc FRC y PL, NIA, R1FSD, R2FSD, R3FSD y en el padrón IV (15 \%) fue R1Pr, R2Pr, TcFRC, PL, R1FSD, NIA y R2FSD. En el patrón tipo I B, el primer músculo en recibir ramos por parte del nervio mediano en el antebrazo fue el pronador redondo, quien en un $53 \%$ de los casos se originó desde un tronco común. El músculo palmar largo es el segundo en ser inervado, dejando en un tercer lugar al flexor radial del carpo, los que en $50 \%$ fueron inervados por un tronco común. El último músculo en ser inervado es el flexor superficial de los dedos, que recibió dos ramos en el 57 \% de los casos. Los resultados son un aporte al conocimiento de la inervación de los músculos del plano superficial del compartimiento anterior del antebrazo.

PALABRAS CLAVE: Anatomía; Antebrazo; Nervio Mediano; Ramos motores.

\section{INTRODUCCIÓN}

En virtud de la importancia de la actividad muscular del antebrazo es necesario conocer detalladamente a los nervios terminales del plexo braquial, tanto en su trayecto como en la distribución de sus diferentes ramos musculares dentro de los compartimientos del antebrazo. Dentro de estos nervios, el nervio mediano es de gran importancia ya que tiene una profusa distribución destinada principalmente al antebrazo y a la mano.

Pocos estudios han descrito un patrón común sobre los puntos de origen o de penetración de estos ramos en los músculos del antebrazo, lo que, presenta un obstáculo a la hora de realizar procedimientos invasivos como las transfe- rencias nerviosas, bloqueos nerviosos o la aplicación de anestésicos locales. Otros tipo de procedimientos que se ven comprometidos por poca información, son los de carácter no invasivos, entre los que destaca la estimulación eléctrica de los puntos motores, método terapéutico utilizado frecuentemente en la rehabilitación física de pacientes con lesiones neuromusculares (Liu et al., 1997; Olave et al., 2002; Lepage et al., 2005; Hwang et al., 2007).

En base a los antecedentes expuestos, el estudio tuvo por objetivo establecer el número de ramos motores, determinar el origen desde el punto de vista biométrico y la distribución secuencial de éstos, en la región proximal del antebrazo.

* Departamento Ciencias Morfológicas, Facultad de Ciencia, Universidad San Sebastián, Concepción, Chile.

** Universidad Estadual de Ciencias da Saude de Alagoas, Maceió, Brasil.

${ }^{* * *}$ Facultad de Medicina, Universidad de La Frontera, Temuco, Chile. 
Con los resultados obtenidos se espera proporcionar un mayor conocimiento acerca de estos ramos en individuos brasileños, comparando estos datos con los mencionados en la literatura internacional, lo que sin duda, será de importancia para la anatomía quirúrgica de la región antebraquial proximal.

\section{MATERIAL Y MÉTODO}

En el estudio se utilizaron 30 miembros superiores, de individuos adultos brasileños, de la Facultad de Medicina de la Universidad Estadual de Ciencias da Saúde de Alagoas, Maceió, Brasil. Estos cadáveres se encontraban fijados en una solución con formaldehído al $10 \%$. De la totalidad de miembros, 16 eran del lado derecho y 14 eran del lado izquierdo.

Esta investigación se formuló bajo una metodología de carácter cuantitativo, transeccional y descriptivo, para lo cual se procedió a realizar una detallada disección de la fosa cubital y compartimento anterior del antebrazo con material quirúrgico "ad-hoc". Se determinó el origen de cada ramo, si eran independientes o provenían de un tronco común. Adicionalmente se registró la distancia de origen respecto a una línea horizontal trazada entre los dos epicóndilos medial y lateral del húmero (LBE), que fue uno de los puntos de referencia seleccionados para las mediciones cuantitativas. El punto de referencia distal fue entre los procesos estiloides radial y ulnar, la Línea Biestiloidea (LBEst), que permitió determinar la localización del origen de los ramos en relación a la distancia LBE-LBEst.

Para patronizar los ramos destinados a los músculos superficiales del compartimiento anterior del antebrazo se consideró el origen de éstos, respecto a una secuencia de localización de proximal a distal. De esta forma se agrupó en 4 patrones, I, II, III y IV, donde el primero se dividió en 2 subtipos.

\section{RESULTADOS}

Las observaciones y registros métricos realizados permitieron agrupar y clasificar de acuerdo a la secuencia de inervación muscular, desde proximal a distal, a los ramos originados desde el nervio mediano y dirigidos a los músculos superficiales del antebrazo, considerándose 4 patrones (Fig. 1).

Patrón Tipo I: Este patrón se presentó en el 70 \% de los casos (19) y se caracterizó por presentar una secuencia de origen de ramos que comenzó con el o los ramos destinados al músculo Pr seguido de los ramos que se dirigieron a los mús- culos PL y FRC. A esto se suma al hecho de que en el $100 \%$ de los casos, los ramos que inervaron al músculo FSD surgieron en un punto distal al origen del nervio interóseo anterior.

En este grupo se establecieron dos subtipos, que se asoció a que los ramos para los músculos Pr, PL y FRC surgieron de forma independiente desde el nervio mediano o presentaron un tronco común (Tc), que se relacionó a los ramos destinados al Pr o a los ramos para los músculos FRC y PL.

Patrón Tipo I A: Este subtipo se presentó en 8 de los miembros examinados $(29 \%)$ y la secuencia de origen describió como primer ramo, aquel que inervó al músculo Pr, el cual se originó en promedio a $13,74 \mathrm{~mm}$, distal a la LBE. A esto se agrega que en el $50 \%$ de estos casos los ramos del músculo se originaron desde un tronco común, el cual surgió en promedio a $14,93 \mathrm{~mm}$, proximal a la LBE.

Siguiendo su trayecto habitual en la fosa cubital, el nervio mediano entregó como segundo ramo, uno dirigido al músculo PL, el que surgió a 18,71 mm, distal a la LBE. El tercer ramo se dirigió hacia el músculo FRC, originándose a un promedio de 29,61 $\mathrm{mm}$ distal a la LBE.

En este patrón, el nervio interóseo anterior surgió previo al origen del primer ramo para el músculo FSD, a un promedio de 53,92 mm distal a la LBE. En cambio, el R1 para el músculo FSD se originó a una distancia promedio de 64,45 mm y el R2 para este músculo se originó en los 4 casos en que estuvo presente, a un promedio de 153,91 mm, distal a la LBE (Fig. 2).

Patrón Tipo I B: Este subtipo consideró la presencia de al menos un Tc que generó ramos para los músculos Pr, FRC y/ o PL. Este patrón de distribución se presentó en el 41 \% de los casos (11 miembros).

En esta distribución, el primer músculo inervado también fue el Pr, en la cual, de los 11 casos incluidos en este patrón, siete de ellos presentaron dos ramos, los que surgieron desde un Tc que se originó desde el nervio mediano, a $10,93 \mathrm{~mm}$ proximal a la LBE, por lo que a este Tc se le consideró como parte de la distribución del nervio mediano en el brazo. El R1 para el músculo Pr se originó en promedio a 5,96 $\mathrm{mm}$ distal a la LBE. El R2 se originó a $6,81 \mathrm{~mm}$, distal a la línea mencionada (Fig. 3A).

Otro elemento característico de esta distribución es que en la totalidad de los casos incluidos en este patrón, los ramos para los músculos FRC y PL se originaron desde un Tc, el cual surgió a 19,70 mm distal a la LBE. El punto de división en ramos para los músculos mencionados se realizó a 27,88 mm distal a esta línea. 


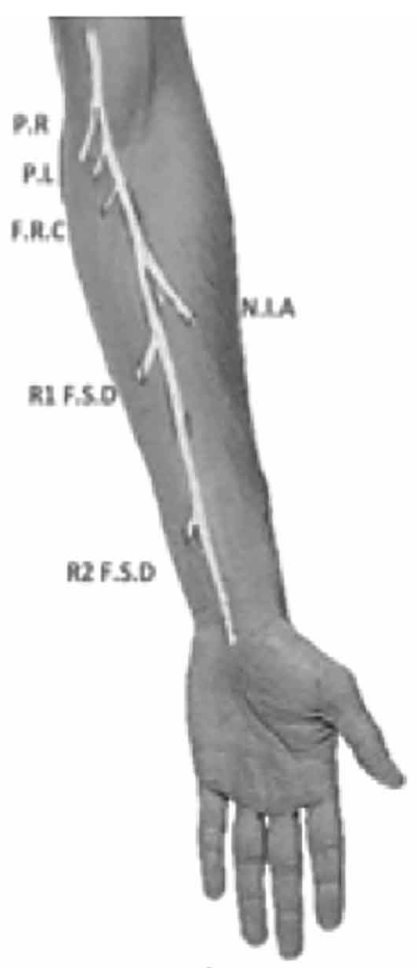

PATRÓNI $70 \%$



PATRON II $4 \%$

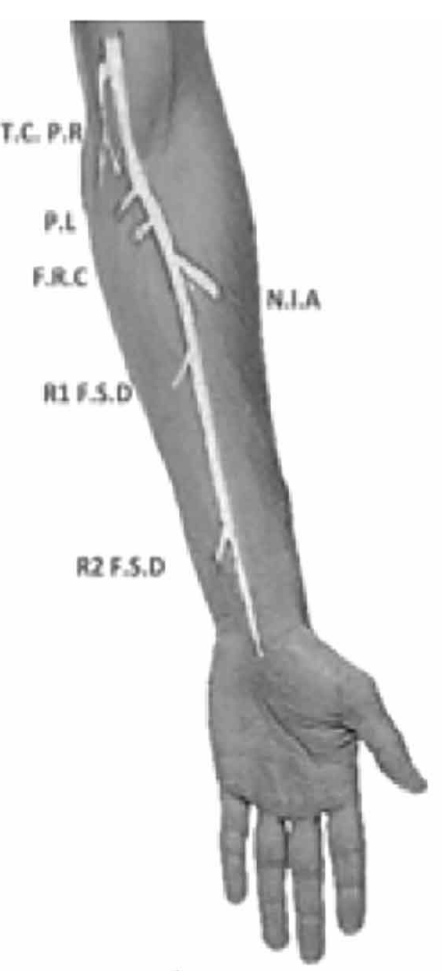

PATRÓN IA $29 \%$

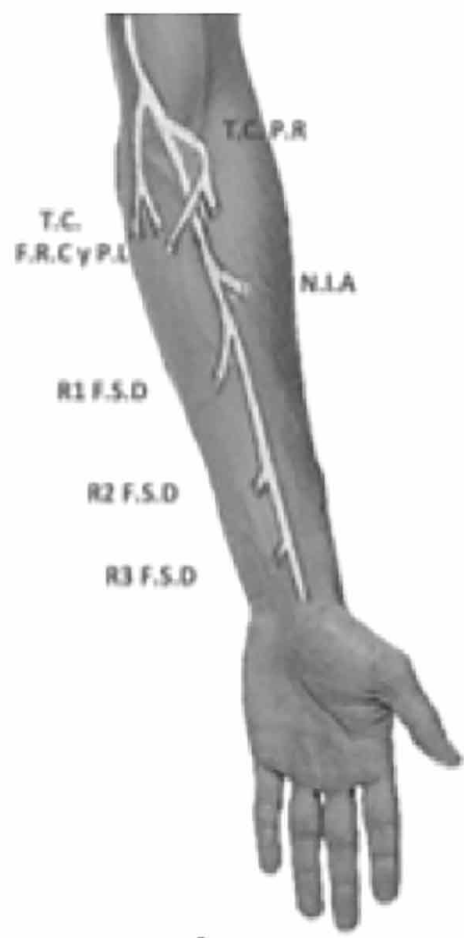

PATRÓN III $11 \%$
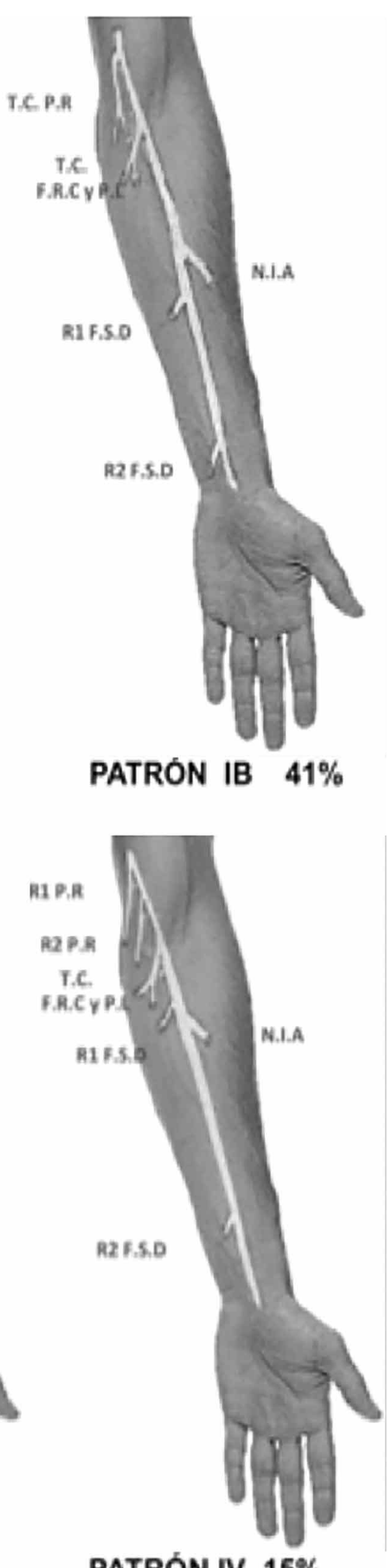

PATRÓN IV $15 \%$

Fig. 1. T.C: Tronco Común - P.R: M. Pronador redondo - P.L: M. Palmar largo - F.R.C: M. Flexor radial del carpo F.S.D: Flexor superficial de los dedos - N.I.A: Nervio interóseo anterior. - R1: Ramo 1 - R2: Ramo 2 - R3: Ramo 3. 


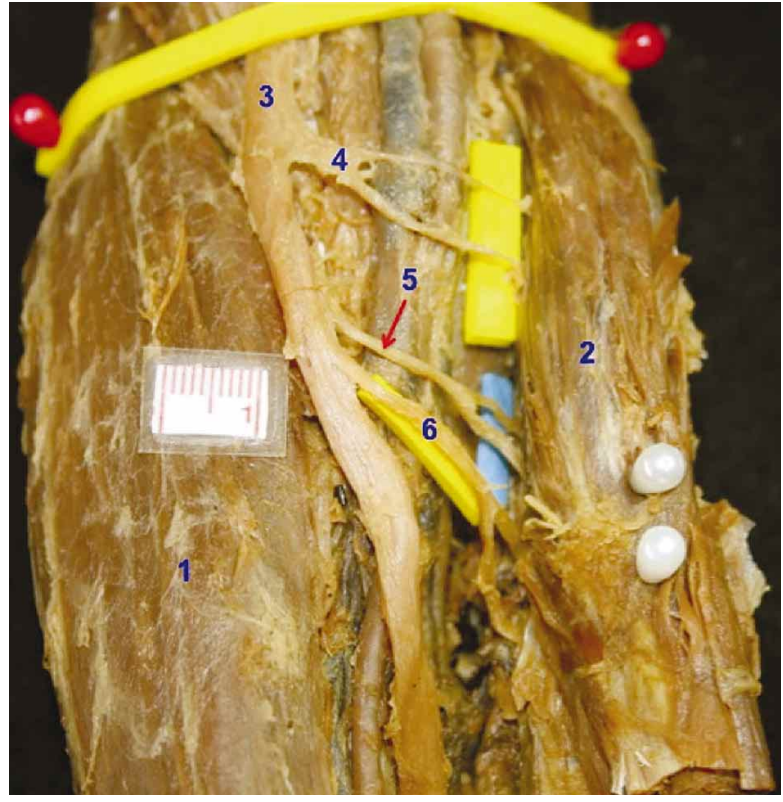

Fig. 2. Patrón I A: 1: Músculo Braquiorradial, 2: Músculo Pronador Redondo, 3: Nervio mediano, 4: Ramo motor del músculo pronador redondo, 5: Ramo motor del músculo palmar largo, 6: Ramo motor del músculo flexor radial del carpo.
Una característica común con el patrón I A, es que el nervio interóseo anterior surgió previo al origen del primer ramo para el músculo FSD. En los 11 casos de este patrón, el nervio interóseo anterior se originó en promedio a 46,60 $\mathrm{mm}$ distal a la LBE, mientras que el R1 para el FSD se originó a 64,04 mm, distal a la línea de referencia.

En la totalidad de los casos analizados, existió un R2 para el músculo FSD, el cual se originó en promedio a 128,18 mm distal a la LBE (Fig. 3B).

Patrón Tipo II: Esta distribución sólo se presentó en un caso, equivalente al $4 \%$ de la muestra. La secuencia se inició con el origen de un Tc que otorgó ramos al músculo Pr. Este Tc surgió a $34 \mathrm{~mm}$ proximal a la LBE, y en la fosa cubital entregó 3 ramos para el mencionado músculo. Este tronco también emitió un ramo para el músculo FRC. En secuencia, el ramo para el músculo PL surgió independiente a $16,46 \mathrm{~mm}$ distal a la LBE.

Este patrón mantuvo la característica de que el nervio interóseo anterior surgió previo al origen de los tres ramos del FSD. El nervio interóseo anterior surgió a $36,59 \mathrm{~mm}$ distal a la LBE, en cambio, los tres ramos del músculo FSD se originaron a $58,71 \mathrm{~mm}, 113,12 \mathrm{~mm}$ y $170,52 \mathrm{~mm}$, respectivamente (Fig. 4).

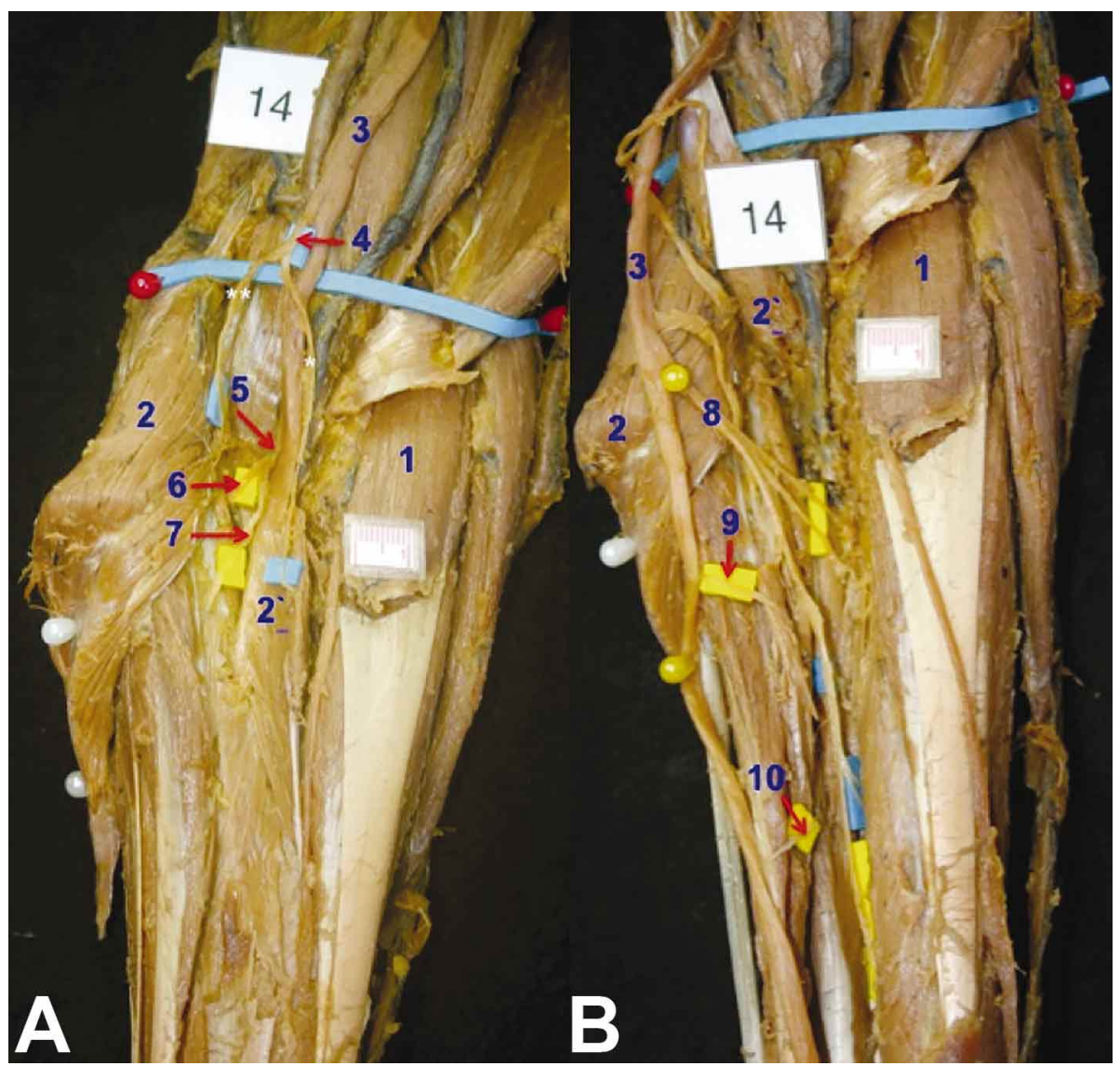

Fig. 3. Patrón I B: 1: Músculo Braquiorradial, 2: Músculo Pronador Redondo, cabeza humeral, 2: Músculo Pronador Redondo, cabeza ulnar, 3: Nervio mediano, 4: Tronco común de los ramos motores para el músculo pronador redondo, R1 (**), y R2 (*), 5: Tronco común para los ramos motores del palmar largo y flexor radial del carpo, 6: Ramo motor del músculo palmar largo, 7: Ramo motor del músculo flexor radial del carpo, 8: Nervio interóseo anterior, 9: Ramo motor R1 para el músculo flexor superficial de los dedos, 10: Ramo motor R2 para el músculo flexor superficial de los dedos. 
Patrón Tipo III: Este tipo se presentó en tres casos (11\%), diferenciándose de los 24 casos restantes por el hecho de que la secuencia de ramos comenzó con la inervación del músculo PL. En dos de los tres casos incluidos en este patrón, este ramo para el músculo PL se originó desde un Tc que fue compartido con el R1 destinado al músculo FRC. La distancia promedio de origen de este Tc fue de 16,79 mm distal a la LBE, mientras el R1 para el músculo PL se originó a 28,48 mm distal a la LBE (Fig. 5).

El otro músculo inervado por el Tc fue el FRC, cuyo ramo se originó a 30,39 mm distal a la LBE.

En dos casos de este patrón, el músculo Pr fue el tercer músculo en recibir ramos desde el nervio mediano. El origen del ramo fue a $30,10 \mathrm{~mm}$ distal a la LBE.

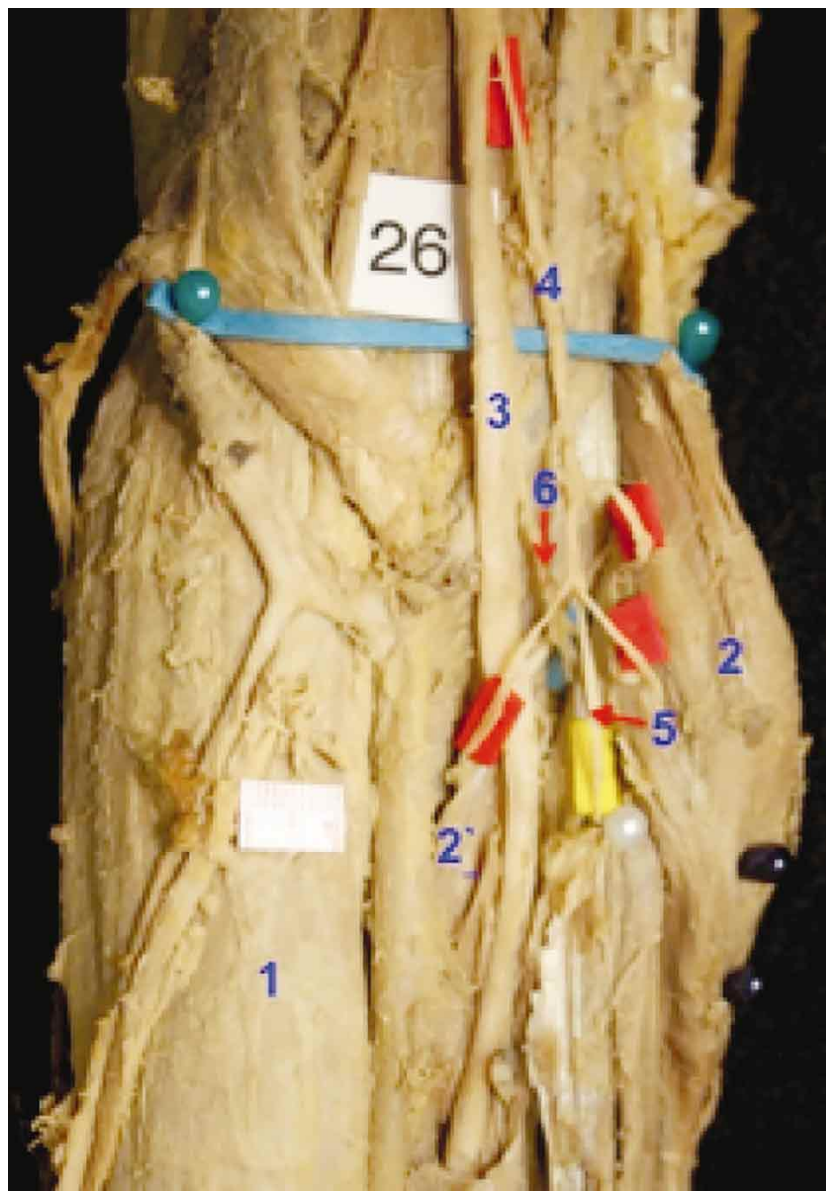

Fig. 4. Patrón II: 1: Músculo Braquiorradial, 2: Músculo Pronador Redondo, cabeza humeral, 2: Músculo Pronador Redondo, cabeza ulnar, 3: Nervio mediano, 4: Tronco común de los ramos motores para el músculo pronador redondo, 5: Ramo motor del músculo flexor radial del carpo, 6: Ramo motor para el músculo palmar largo.
En este patrón al igual que los I A, I B y II, el nervio interóseo anterior se originó proximal a los ramos del músculo FSD. El nervio interóseo anterior se originó a un promedio de $51 \mathrm{~mm}$ distal a la LBE, en cambio, los tres ramos motores del músculo FSD se originaron en promedio a 64,21 $\mathrm{mm}, 103,73 \mathrm{~mm}$ y $175,54 \mathrm{~mm}$, respectivamente.

Patrón Tipo IV: Se presentó en cuatro casos (15\%) y su característica principal fue que el primer ramo para el músculo FSD se originó en un punto proximal al origen del nervio interóseo anterior.

En los cuatro casos, el primer ramo que se originó desde el nervio mediano fue para el músculo $\mathrm{Pr}$, que se originó a un promedio de $6,90 \mathrm{~mm}$ proximal a la LBE. En tres casos este músculo recibió dos ramos y su R2 se originó en promedio a $8,53 \mathrm{~mm}$ proximal a la referida línea.

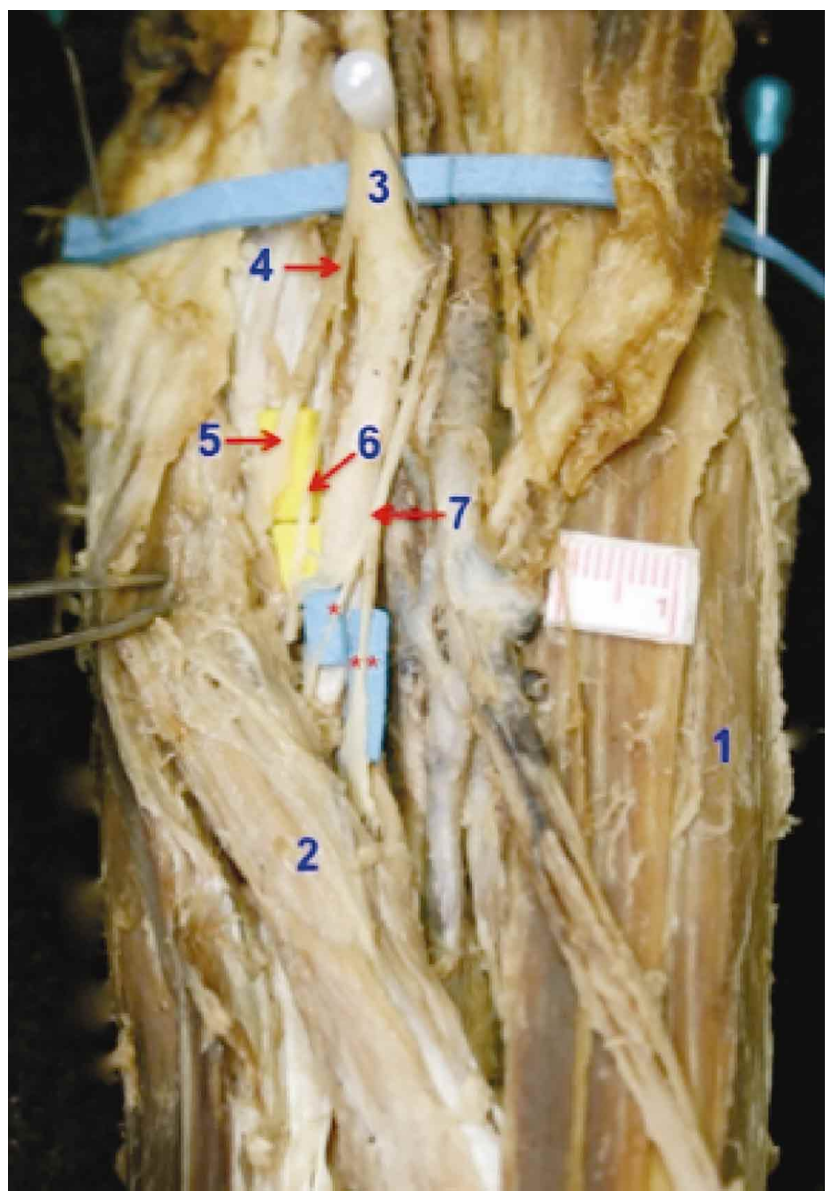

Fig. 5. Patrón III: 1: Músculo Braquiorradial, 2: Músculo Pronador Redondo, 3: Nervio mediano, 4: Tronco común para los ramos motores del palmar largo y flexor radial del carpo, 5: Ramo motor del músculo palmar largo, 6: Ramo motor para el músculo flexor radial del carpo, 7: Tronco común de los ramos motores para el músculo pronador redondo, R1 (*) y R2 (**). 
El segundo músculo inervado por el nervio mediano fue el PL, cuyo ramo se originó desde un Tc. En un 75 $\%$ de los casos este tronco emitió también un ramo para el músculo FRC. El origen de este Tc se localizó a 27,59 $\mathrm{mm}$ distal a la LBE y el origen del ramo para el PL fue a $31,19 \mathrm{~mm}$ distal a la mencionada línea.

En dos de estos casos, el tercer músculo inervado fue el FRC, y en los dos casos restantes fue el músculo FSD. En la totalidad de los casos el ramo destinado al músculo flexor radial del carpo se originó desde un tronco común, en tres de los casos compartió el Tc con el músculo PL y el caso restante el Tc fue compartido con el R1 del músculo FSD. El origen de los ramos destinados al músculo FRC fue en promedio a 32,95 $\mathrm{mm}$ distal a la LBE.



Fig. 6. Patrón IV: 1: Músculo Braquiorradial, 2: Músculo Pronador Redondo, 3: Nervio mediano, 4: Ramo motor R1 para el músculo pronador redondo, 5: Ramo motor R2 para el músculo pronador redondo, 6: Ramo motor del músculo palmar largo, 7: Ramo motor R1 para el músculo flexor superficial de los dedos, 8: Ramo motor para el músculo flexor radial del carpo, 9: Nervio interóseo anterior.
Una característica distintiva de este patrón fue que en los cuatro casos, el R1 para el FSD surgió proximal al origen del nervio interóseo anterior, a una distancia promedio de 39,88 $\mathrm{mm}$ distal a la LBE, en cambio el nervio interóseo anterior se originó en promedio a 40,63 mm, distal a la misma línea. A esto se agrega que en el $75 \%$ de los casos el R1 para el músculo FSD surgió desde un Tc, originado a 23,28 mm distal a la LBE (Fig. 6). En el $50 \%$ de los casos sólo se presentó un ramo (R1), dejando a los dos casos restantes, uno con dos (R1 y R2) y el otro con tres (R1, R2 y R3).

\section{DISCUSIÓN}

La inervación de los músculos de la región anterior del antebrazo es dada principalmente por el nervio mediano, participando parcialmente el nervio ulnar. El lugar específico del origen de los ramos motores para los músculos del compartimiento anterior del antebrazo son variables, por lo que se hace necesario conocer la secuencia de origen de éstos y registrar su localización desde el punto de vista biométrico.

Con respecto a la presencia de ramos motores en el brazo, Testut \& Latarjet (1972), Williams et al. (1998); Drake et al. (2005) y Rouvière \& Delmas (2005), describieron la presencia de un ramo para el músculo pronador redondo en el tercio distal del brazo en $37 \%$ de los casos, disposición con la que concordamos. Este porcentaje es muy inferior al señalado por Alves et al. (2004), en que este ramo motor estuvo en más del $90 \%$ de su muestra.

La descripción realizada por Sappey (1889) y Poirier \& Charpy (1902), en la que señalaron que la forma en la que se originan los ramos desde el nervio mediano en la mitad proximal del antebrazo, puede ser de forma independiente $o$ a través de uno o dos troncos principales, también se vio expresada en nuestra investigación.

Autores como Cruveilhier (1851); Testut \& Latarjet; Sinelnikov (1986); Latarjet, \& Ruiz Liard (2004) y Rouvière $\&$ Delmas, describieron la presencia de un ramo para la musculatura de la capa superficial del compartimiento anterior del antebrazo, del cual surgen ramos para los cuatro músculos que conforman esta capa. En nuestro estudio se encontró un solo caso que coincide con esta descripción, en cambio, la distribución más frecuente (77 \%) se asoció a la presencia de uno o dos troncos comunes, en donde uno de ellos estuvo formado por los ramos para el músculo pronador redondo y el tronco restante fue común para los ramos de los músculo flexor radial del carpo y palmar largo. 
Al considerar la inervación de cada uno de los cuatro músculos de este plano superficial, se observó que el primer músculo inervado fue el pronador redondo $(87 \%)$ al igual que en el estudio de Sunderland \& Ray (1921) y que también coincide con Gunther et al. (1992), que señalaron que un $95 \%$ de sus casos (20), el primer ramo era destinado al músculo mencionado.

$\mathrm{Al}$ analizar el número de ramos para el pronador redondo, el intervalo de cantidad y el promedio de ramos encontrados en nuestro estudio coincide con Alves et al., ya que en su serie de 18 miembros superiores el número de ramos varió entre uno y tres con un promedio de 1,8.

Por su parte, Fuss \& Wurzl señalaron que el $44 \%$ de los casos presentó dos ramos, seguido de un $28 \%$ con un ramo, cifras similares a nuestra investigación, ya que en el 53 $\%$ de casos se presentaron dos ramos y en el $30 \%$ sólo presentó un ramo. Sin embargo ambas cifras difieren de los planteado por Lepage et al. que señalaron que el $87 \%$ de los 30 casos de su muestra presentaron dos ramos para este músculo. A diferencia de lo anterior, Linell (1921) describió la presencia de un solo ramo para el músculo pronador redondo, cuyo origen fue independiente, dato que difiere a nuestra investigación, ya que el $53 \%$ de los casos presentó dos ramos motores (R1 y R2), dentro de los cuales, en el $69 \%$ de los casos, estos ramos surgieron desde un tronco común.

A pesar de esto, en ambos estudios, a los que se suma Gunther et al. (1993), el punto de origen promedio quedó establecido entre 1 a $2 \mathrm{~cm}$, distal a la LBE, a lo que se agrega el hecho de que el punto de origen que se analiza presentó una gran variabilidad, el cual para Linell, se extendió hasta $4 \mathrm{~cm}$, proximal a la LBE. Al respecto, nuestro estudio concuerda con lo señalado por Linell, ya que al cuantificar esta variabilidad se encontraron casos en la cual este intervalo se extendió entre los 3,6 cm proximal y $\operatorname{los} 6,25 \mathrm{~cm}$ distal a la L.B.E.

En el estudio realizado por Canovas et al. (1998), se comparte la descripción respecto de la presencia de dos ramos para el músculo pronador, siendo el primero de ellos denominado ramo proximal, el cual surgió de forma independiente en un $80 \%$ de los casos del citado estudio, dato que supera al $31 \%$ que se obtuvo en nuestra muestra. A esto se sumó que el promedio de la distancia de origen de nuestro estudio (1,75 cm distal al la LBE) superó los $0,17 \mathrm{~cm}$, distal a la LBE, señalado por estos autores.

Respecto a la presencia de un ramo distal para el músculo pronador redondo, fue el mismo estudio de Canovas et $a l$, que describieron la presencia de este ramo distal, indicando que dicho ramo se originó de forma independiente en el $30 \%$ de los casos, siendo más frecuente que este ramo se origine de forma común con otros ramos destinados a los músculos superficiales del compartimiento anterior del antebrazo. Esto mismo se observó en nuestra investigación, ya que en el $29 \%$ de los 21 casos que presentaron este ramo, éste se originó de forma independiente.

En el caso de los tres restantes músculos del plano superficial del compartimiento anterior del antebrazo, Linell señaló que estos tres músculos fueron inervados por un tronco común, sin embargo, en nuestra investigación esta característica se presentó en un solo caso, siendo el formato de origen más frecuente, el que se asoció a la presencia de un tronco común para los músculos flexor radial del carpo y palmar largo $(50 \%)$. A esto se agrega el hecho de que estos mismos músculos fueron inervados por ramos independientes en al menos el $36 \%$ de los casos, siendo esto mismo aplicado al músculo flexor superficial de los dedos, ya que en un $87 \%$ de los casos, este músculo fue inervado por ramos que surgieron de forma independiente desde el nervio mediano. Esta misma diferencia se encontró al comparar nuestro estudio con los de Fuss \& Wurzl y Gunther et al. (1992), quienes establecieron que los ramos para estos tres músculos epicondilares mediales se originaron de un tronco común entre un 50 y $56 \%$ de los casos. Fuss \& Wurzl describieron la presencia de dos ramos en un $40 \%$ y en tres en un $4 \%$. Otro detalle de la muestra de Gunther et al. (1992) fue que se observaron tres casos en donde este grupo de ramos no existió, dejando la inervación de estos músculos al nervio interóseo anterior.

En nuestra muestra, un $4 \%$ de los casos presentó un tronco común para los tres músculos en análisis, en cambio, el $62 \%$ presentó dos ramos, siendo uno de ellos, un tronco común para los músculos flexor radial del carpo y palmar largo y el $34 \%$ restante de los casos presentaron tres ramos.

Nuestras observaciones coinciden con el estudio de Canovas et al., quienes señalaron que los ramos destinados a los músculos palmar largo y flexor radial del carpo, se desprendieron de forma conjunta en un $50 \%$ de su muestra. Otra semejanza es la distancia promedio de origen que se estableció en 2,5 cm, distal a la LBE.

Si se analiza por separado estos tres músculos, podemos establecer que en un $86 \%$ de los casos el palmar largo fue el segundo músculo en recibir ramos desde el nervio mediano, dejando en tercer lugar al flexor radial del carpo, para distalmente entregar ramos para el flexor superficial de los dedos.

En base a esta distribución resulta importante destacar que en el $7 \%$ de la muestra (dos casos), el músculo palmar largo estuvo ausente, agenesia que varió drásticamente la secuencia de ramos que entregó el nervio mediano. 
Liu et al., analizaron el número de ramos para el músculo palmar largo, y establecieron que este músculo recibió un sólo ramo en el $100 \%$ de los casos, igual a lo observado en nuestra serie.

Blair \& Joos (1982), señalaron que en un $95 \%$ de los casos el músculo flexor radial del carpo fue inervado por solo un ramo, semejante a nuestro resultado $(97 \%)$. Esta misma coincidencia se da también con Sunderland \& Ray, los que establecieron que este músculo estuvo inervado por un solo ramo en el $90 \%$ de sus 20 casos. Sin embargo, si nos enfocamos en la forma de origen, Sunderland \& Ray, indicaron que en un $20 \%$ de los casos, el ramo para el flexor radial del carpo presentó un origen independiente y en el $35 \%$ de la muestra presentó un origen común con el m. flexor superficial de los dedos, en cambio, en nuestro estudio, el $38 \%$ de los ramos para este músculo surgieron de forma aislada, estableciendo como formato de origen más frecuente el que presenta un tronco que fue común con el ramo del músculo palmar largo, el cual se presentó en el $83 \%$ de los casos que surgieron de forma compartida con otro(s) ramo(s).

En el $87 \%$ de los casos el músculo flexor superficial de los dedos fue el cuarto músculo de este plano superficial en recibir ramos, dato que coincide con lo expuesto por Gunther et al. (1992) quienes además de ubicar a este músculo en el mismo lugar de secuencia, estableció que en el $75 \%$ de los casos este músculo presentó un solo ramo, disposición que supera al $26 \%$ de nuestra investigación. En el $57 \%$ de nuestra serie, se presentaron dos ramos para este músculo, disposición inferior al $83 \%$ de casos que presentaron Lepage et al.

Para Linell y Canovas et al. aproximadamente en un $50 \%$ se identificaron ramos adicionales para el músculo flexor superficial de los dedos, los cuales emergieron del nervio mediano en el tercio distal del antebrazo. En relación a este dato, Cruveilhier, Rauber (1898), Poirier \& Charpy y Rouvière \& Delmas, señalaron que independientemente de los ramos que inervan la parte proximal de este músculo, el nervio mediano origina para este músculo dos o tres ramos adicionales, que se desprenden gradualmente del nervio mediano en su trayecto dentro del antebrazo, disposición que se presentó en el $74 \%$ de los miembros superiores analizados en nuestra investigación.

Williams et al., agregaron que estos ramos adicionales se relacionan con el vientre muscular que tiene el flexor superficial de los dedos para el dedo índice, descripción que coincide con lo observado en todas las muestras de nuestra serie para los ramos R2 y R3. Dicha descripción difiere con Llusá et al. (2013) quienes señalaron que los ramos musculares que surgen del nervio mediano en la fosa cubital no van destinados al músculo flexor superficial de los dedos.
En el estudio de Sunderland \& Ray se señala que este músculo flexor superficial de los dedos también recibió ramos después de originados los ramos para los otros músculos de este plano superficial. A esta descripción se sumó la gran variabilidad en lo que respecta a la cantidad de ramos que penetraron a este músculo, ya que en el $35 \%$ de los casos fue inervado por dos ramos, dejando al $65 \%$ restante como aquellos que recibieron entre tres y siete ramos. Al respecto, nuestra investigación coincide en lo que concierne al número de ramos, pero difiere del intervalo de ramos que penetraron este músculo, ya que en nuestra muestra el intervalo se extendió entre uno y tres ramos.

Con relación al origen de los ramos para este músculo, Sunderland \& Ray señalaron que en el $70 \%$ de los casos, se originaron del nervio mediano, dejando al $30 \%$ restante, como aquellos en donde sus ramos surgieron desde el nervio interóseo anterior, resultado que difiere sustancialmente con el $97 \%$ de nuestros casos, en que el ramo R1 surgió desde el nervio mediano, dejando un solo caso (3\%) como aquel en la cual este ramo se originó desde el nervio interóseo anterior, cifra que se acerca a las planteadas por Liu et al., en que sólo el $10 \%$ de estos ramos surgieron desde el nervio interóseo anterior.

Respecto a la distancia del punto de origen del primer ramo para el músculo flexor superficial de los dedos, Gunther et al. (1992) señalaron que el promedio alcanzó $7,0 \mathrm{~cm}$ distal a la LBE, cifra que para Linell se establece en $10 \mathrm{c} 12 \mathrm{~cm}$ distal a la LBE, ambos promedios resultan mayores que $\operatorname{los} 5,73 \mathrm{~cm}$ distal a la LBE de nuestra investigación, pero superan los 3,5 $\mathrm{cm}$ distal a la LBE que informara Canovas et al.

Sunderland \& Ray también aportaron datos comparables respecto al formato de origen de los ramos para este músculo, en donde en el $75 \%$ de los casos sus ramos surgieron desde un tronco común, siendo lo más frecuente (35\%), un origen común con el músculo flexor radial del carpo; en cambio, en nuestro estudio las cifras señalaron que en el $87 \%$ de los casos el ramo R1 y en el $100 \%$ de los ramos R2 y R3 se originaron de forma independiente. Por último, Sunderland \& Ray, establecieron que en el $95 \%$ de los casos, estos ramos surgieron distal a la LBE, cifra concordante con nuestro $98 \%$.

Los padrones de origen y la distribución mostrada en el presente estudio, permiten conocer con más detalle a los ramos emitidos por el nervio mediano a nivel de la región cubital, considerando una línea transversal de referencia (LBE) para indicar si ellos se originaron proximal o distal a esta línea. Los resultados obtenidos clarifican y aportan detalles anatómicos y biométricos al conocimiento del nervio mediano y los músculos superficiales de la región anterior del antebrazo. 
RIVEROS, A.; SOUSA-RODRIGUES, C. F. \& OLAVE, E. Origin and distribution of the motor branches for the superficial muscles of the anterior region of the forearm in Brazilian individuals. Int. J. Morphol., 34(1):356364, 2016.

SUMMARY: The median nerve is responsible for the innervation of the muscles of the superficial layer of the anterior compartment of the forearm, it originates along its course in the arm and forearm, branches to these muscles. Some branches originated from common trunks $(\mathrm{Ct})$. We studied 30 upper limbs of Brazilian adults corpses, fixed in $10 \%$ formaldehyde in order to obtain an anatomical and biometric description of the origin and distribution of the branches of the median nerve in the superficial muscles of the forearm: pronator teres $(\mathrm{Pt})$, palmaris longus (PL), flexor carpi radialis (FCR), flexor digitorum superficialis (FDS) and classify these branches ( $\mathrm{Br}$ ) according to their origin. We performed dissections and recorded origins in relation to the biepicondilar line (BEL). With the results we obtained a classification of the branches to the muscles of the superficial layer of the anterior compartment in 4 patterns (I, II, III, IV; the I classified in IA and IB), including the origin of anterior interosseous nerve (AIN). In the pattern IA (29\%), branches originated from proximal to distal in the following sequence: CtPT, PL, FCR, AIN, Br1FDS, Br2FDS; in pattern IB (41\%) showed CtPt, TcFCR and PL, AIN, Br1FDS, Br2FDS; in pattern II (4\%) was CtPt and FCR, PL, AIN, Br1FDS, Br2FDS, Br3FDS; in pattern III (11\%) it was CtPt, Ct FCR and PL, AIN, Br1FDS, Br2FDS, Br3FDS and in pattern IV (15\%) was $\mathrm{Br} 1 \mathrm{Pt}, \mathrm{Br} 2 \mathrm{Pt}, \mathrm{CtFCR}$ and PL, Br1FDS, AIN, Br2FDS. In type pattern IB, the first muscle to receive branches from the median nerve in the forearm was the PT, which in $53 \%$ of cases originated from a common trunk. The PL was the second to be innervated, leaving third place to FCR, which $50 \%$ were supplied by a common trunk. The latest to be innervated muscle was the FDS, which received two branches in $57 \%$ of cases. The results are a contribution to the knowledge of the innervation of the muscles of the superficial layer of the anterior compartment of the forearm.

KEY WORDS: Anatomy; Forearm; Median nerve; Motor branches.

\section{REFERENCIAS BIBLIOGRÁFICAS}

Alves, N.; Cândido, P. L. \& Frazão, R. Innervation of the pronator teres muscle. Int. J. Morphol., 22(3):237-40, 2004.

Blair, W. F. \& Joos, K. The innervation of flexor carpi radialis. An interfascicular dissection. Arch. Neurol., 39(10):647-9, 1982.

Canovas, F.; Mouilleron, P. \& Bonnel, F. Biometry of the muscular branches of the median nerve to the forearm. Clin. Anat., 11(4):239-45, 1998.

Cruveilhier, J. Traite D`Anatomie Descriptive. 4a ed. Paris, Asselin, 1851. pp. 880 .

Drake, R. L.; Vogls, W. \& Mitchell, A. W. M. Gray: Anatomía para Estudiantes. Madrid, Elsevier España, 2005. pp.1058.

Fuss, F. K. \& Wurzl, G. H. Median nerve entrapment. Pronator teres syndrome. Surgical anatomy and correlation with symptom patterns. Surg. Radiol. Anat., 12(4):267-71,1990.

Gunther, S. F.; DiPasquale, D. \& Martin, R. The internal anatomy of the median nerve in the region of the elbow. J. Hand Surg., 17(4):648-56, 1992.

Gunther, S. F.; DiPasquale, D. \& Martin, R. Struthers' ligament and associated median nerve variations in a cadaveric specimen. Yale J. Biol. Med., 66(3):203-8, 1993.
Hwang, K.; Jin, S.; Hwang, S. H.; Lee, K. M. \& Han, S. H. Location of nerve entry points of flexor digitorum profundus. Surg. Radiol. Anat., 29(8):617-21, 2007.

Latarjet, M. \& Ruiz Liard, A. Anatomía Humana. Vol. 1. $4^{\mathrm{a}}$ ed. Buenos Aires, Médica Panamericana, 2004. pp.896.

Lepage, D.; Parratte, B.; Tatu, L.; Vuiller, F. \& Monnier, G. Extra- and intramuscular nerve supply of the muscles of the anterior antebrachial compartment: applications for selective neurotomy and for botulinum toxin injection. Surg. Radiol. Anat., 27(5):420-30, 2005.

Linell, E. A. The distribution of nerves in the upper limb, with reference to variabilities and their clinical significance. J. Anat., 55(Pt. 2-3):79-112, 1921.

Liu, J.; Pho, R. W.; Pereira, B. P.; Lau, H. K. \& Kumar, V. P. Distribution of primary motor nerve branches and terminal nerve entry points to the forearm muscles. Anat. Rec., 248(3):456-63, 1997.

Llusá Pérez, M.; Palazzi Coll, S. \& Valer Tito, A. Anatomía Quirúrgica del Plexo Braquial y Nervios Periféricos de la Extremidad Superior. Buenos Aires, Médica Panamericana, 2013. pp.374.

Olave, E.; Gabrielli, C.; Braga, M. T. T. \& del Sol, M. Biometric aspects of the motor branches of the musculocutaneous nerve to the brachial muscle. Rev. Chil. Anat., 20(2):231-6, 2002.

Poirier, P. \& Charpy, A. Traité d'Anatomie Humaine. Vol. 3. 2 $2^{\mathrm{a}}$ ed. Paris, Masson et Cie, 1902. pp.498.

Rauber, A. Lehrbuch der Anatomie des Menschen. Vol. 2. $5^{\text {a }}$ ed. Leipzig, Verlag von Arthur Georgi, 1898. pp.882.

Rouvière, H. \& Delmas, A. Anatomía Humana: Descriptiva, Topográfica y Funcional. Vol. 3. 11 a ed. Barcelona, Masson, 2005. pp. 736.

Sappey, P. C. Traíte D`Anatomie Descriptive. Vol. 3. $4^{\text {a }}$ ed. Paris, Lecrosnier et Babé, 1889. pp.835.

Sinelnikov, R. D. Atlas de Anatomía Humana. Vol. 3. 4ª ed. Moscú, MIR, 1986. pp.397.

Sunderland, S. \& Ray, L. J. Metrical and non-metrical features of the muscular branches of the median nerve. J. Comp. Neurol., 85(2):191-203, 1921.

Testut, L. \& Latarjet, A. Tratado de Anatomía Humana. Vol. 3. $9^{\mathrm{a}}$ ed. Barcelona, Salvat, 1972. p.1142.

Williams, P. L.; Bannister, L. H.; Berry, M. M.; Collins, P.; Dyson, M.; Dussek, J. E. \& Ferguson, M. W. J. Anatomía de Gray. Bases Anatómicas de la Medicina y la Cirugía. Vol. 2. $38^{\mathrm{a}}$ ed. Madrid, Churchill LivingstoneHarcourt Brace, 1998. pp.2099.

Dirección para Correspondencia:

Prof. Mg. Andrés Riveros

Depto. de Ciencias Morfológicas

Universidad San Sebastián- Sede Concepción

Lientur 1457 C.P: 4080871

Concepción - CHILE 